\title{
Autosomal juvenile retinoschisis without foveal retinoschisis
}

\author{
KATSUHIRO YAMAGUCHI AND SATOSHI HARA \\ From the Department of Ophthalmology, School of Medicine, Tohoku University, Japan
}

SUMMARY We describe hereditary retinoschisis without foveal retinoschisis in a girl (the product of a consanguineous marriage) and her father. The father had peripheral retinoschisis with dendritic changes of the retina and good visual acuity. The daughter had peripheral retinoschisis complicated by vitreous haemorrhage and retinal detachment. Neither had foveal retinoschisis. The pattern in a scotopic single-flash electroretinogram differentiated this condition from other forms of retinoschisis. We believe that these cases represent the autosomal recessive form of juvenile retinoschisis without foveal retinoschisis.

Autosomal juvenile retinoschisis ${ }^{1}$ is a rare vitreoretinal dystrophy characterised by peripheral retinoschisis without pathognomonic foveal retinoschisis. We describe a father and daughter who had peripheral retinoschisis without foveal retinoschisis, which possibly was transmitted as an autosomal recessive disorder. The results of a scotopic singleflash electroretinogram showed a slightly reduced a

Correspondence to Katsuhiro Yamaguchi, MD, Department of Ophthalmology, School of Medicine, Tohoku University, 1-1, Sieryo-cho, Sendai, Miyagi, 980, Japan. wave, a diminished $b$ wave that returned to above baseline levels, and diminished oscillatory potentials, a pattern considered pathognomonic in this disorder.

\section{Case reports}

CASE 1

A 2-year-old girl suffered a fall on 16 May 1987. Several days later her mother noticed the presence of right esotropia. The child was referred to Tohoku University Hospital because of a vitreous haemorr-

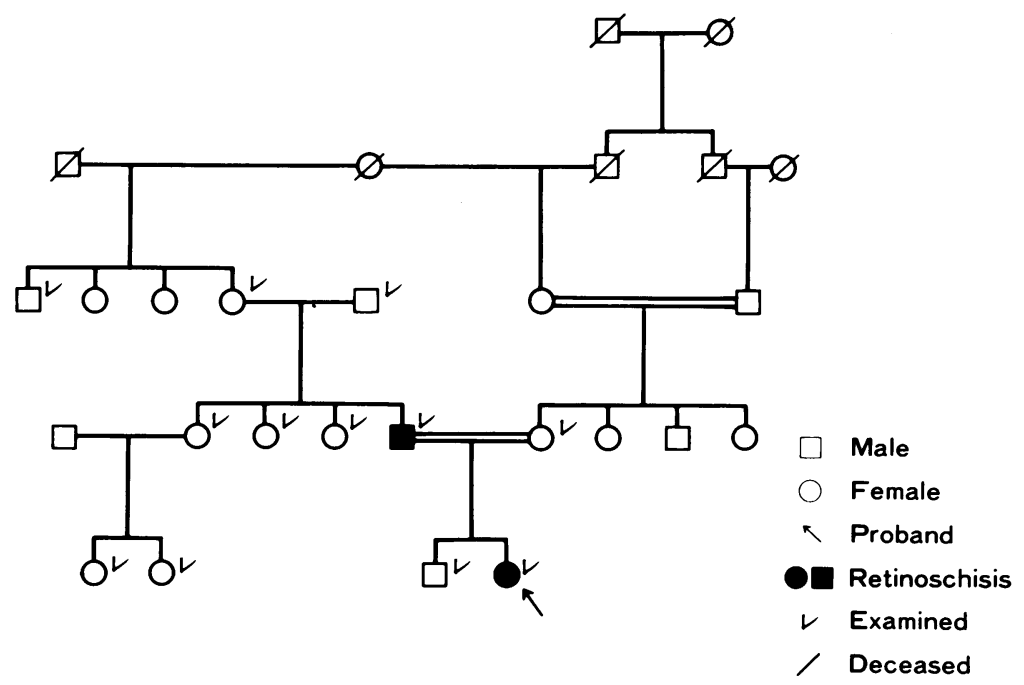

Fig. 1 Pedigree of the affected family. The proband is the product of a consanguineous marriage. 


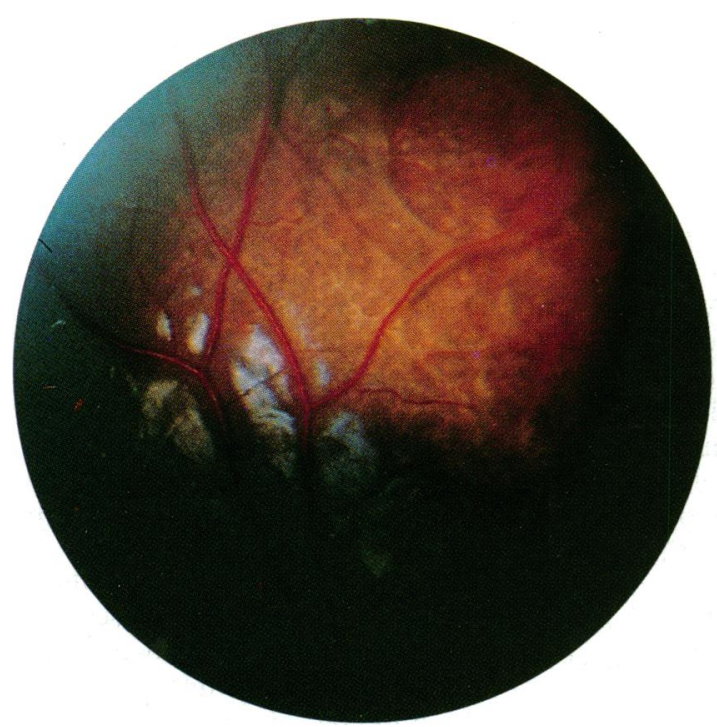

Fig. 2 The left inferotemporal peripheral fundus of the proband shows retinoschisis and a large break in the inner layer of the retina.

hage in the right eye on 27 May 1987. She was the product of an uneventful pregnancy resulting from a consanguineous marriage (Fig. 1). Her physical and mental development had been normal.

The ophthalmic examination showed 15 prism dioptres of right esotropia and normal eye movements. The pupil was normal in shape and showed a normal light reflex. Visual acuity could not be measured because of the patient's youth. The right fundus was difficult to see owing to vitreous haemorrhage. The left fundus showed peripheral retinoschisis with inner retinal holes at the equator (Fig. 2). The macula appeared normal, and no foveal retinoschisis was observed (Fig. 3). A scotopic single-flash electroretinogram, which was recorded after 20 minutes of dark adaptation, displayed a slightly reduced a wave, a diminished $b$ wave that returned to above baseline levels, and diminished oscillatory potentials (Fig. 4).

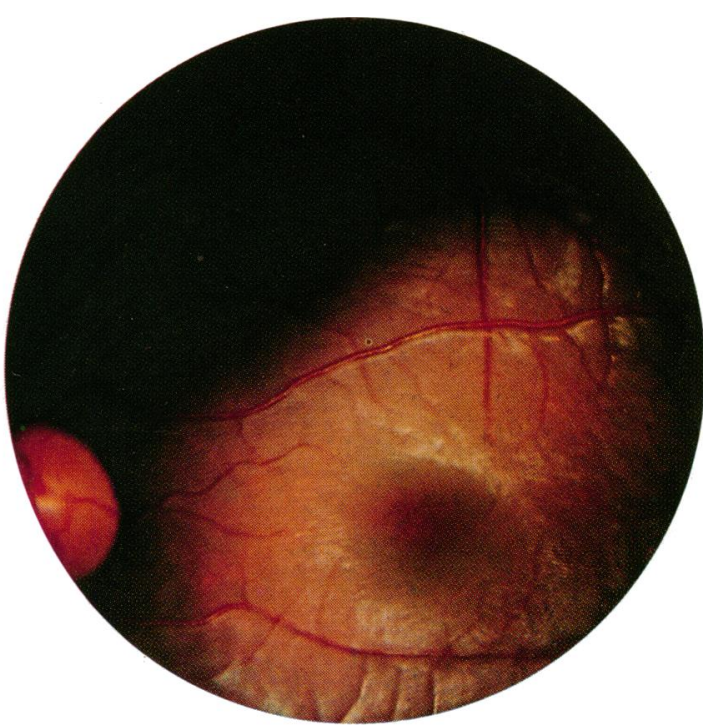

Fig. 3 The left macula of the proband appeared normal, and no foveal retinoschisis is observed.

Ultrasonography disclosed a strong sheet-like echo that was suggestive of a retinal detachment.

After a week's observation an ophthalmoscopic examination of the girl's right eye showed a small, full-thickness retinal detachment that involved the inferotemporal retina and extended from the 5 to 9 o'clock meridian. Also observed were a large grey membrane floating in the inferotemporal vitreous cavity and two holes in the outer retinal layer at the 6 o'clock meridian between the vascular arcade and equator, among the old vitreous haemorrhage. Another lesion of retinoschisis spread across the superotemporal fundus. The optic disc appeared normal, and foveal retinoschisis was not observed.

Surgical treatment consisting of cryoretinopexy and scleral buckling was performed successfully on 9 June 1987. The retina became reattached during the postoperative period of observation.

$$
\text { Control Patient }
$$


Fig. 4 Right: Single-flash electroretinogram, measured after 20 minutes of dark adaptation, shows a normal a wave, a reduced $b$ wave that returned to above the baseline levels, and diminished oscillatory potentials in the proband. Left: $A n$ electroretinogram from a normal 2-year-old, measured after 20 minutes of dark adaptation. 


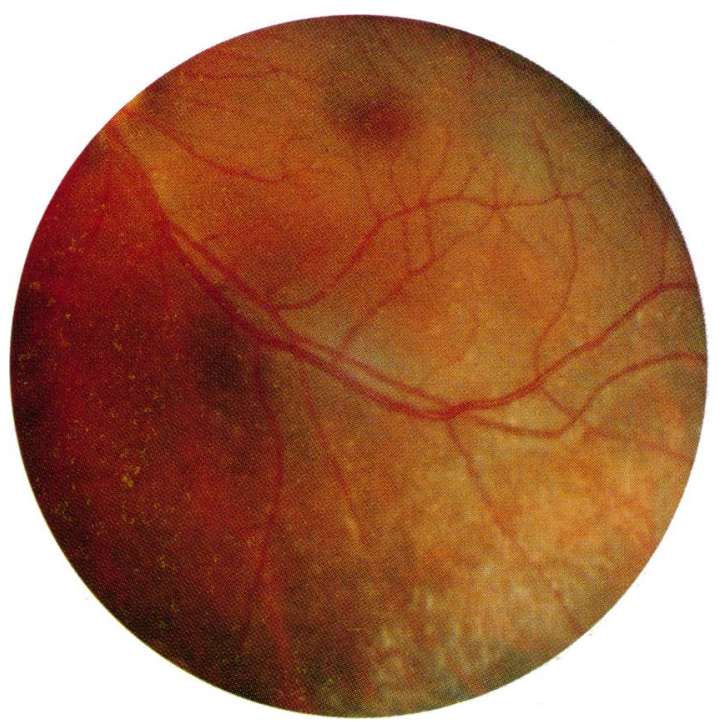

Fig. 5 The left peripheral fundus of the proband's father discloses dendritic filariform structures of the inner retina. The macula appears intact, and foveal retinoschisis is absent.

CASE 2

The 35-year-old father of the proband was examined on 27 May 1987. His best corrected visual acuity was 20/20 in both eyes. Colour differentiation assessed with Ishihara plates, intraocular pressure measured by Goldmann tonometry, and anterior segments examined by slit-lamp biomicroscopy appeared normal in both eyes. Each fundus demonstrated peripheral retinoschisis and dendritic filariform structures in the inner layer of the schisis. No foveal retinoschisis was observed (Fig. 5). Visual field examination by Goldmann perimetry showed absolute depressions in the peripheral fields in both eyes.

A scotopic single-flash electroretinogram, recorded after 20 minutes of dark adaptation, displayed a slightly reduced a wave, a diminished b wave that returned to above baseline levels, and diminished oscillatory potentials (Fig. 6). Fluorescein angiography disclosed arborescent figures of retinal vessels in the regions of retinoschisis.

\section{Discussion}

Among the various hereditary retinoschises foveal retinoschisis is an important pathognomonic finding. In X-linked juvenile retinoschisis, which is carried by the $\mathrm{X}$ chromosome and is transmitted in a recessive manner with a high degree of penetrance, foveal retinoschisis occurs in $98 \%$ to $100 \%$ of patients. In approximately $50 \%$ of patients foveal retinoschisis is the sole pathological finding. ${ }^{2}$ This disease is characterised by a negative electroretinogram, which showed a normal a wave, a b wave that does not return to the baseline values, and reduced oscillatory potentials. ${ }^{3}$ In a female carrier of X-linked juvenile retinoschisis radial wrinkling of the inner limiting membrane occurs around the fovea, but no peripheral retinoschisis is seen. ${ }^{4}$ The electroretinogram is normal. Favre-Goldmann disease,${ }^{5}$ which is inherited in an autosomal recessive manner, is not only characterised by peripheral retinoschisis and foveal retinoschisis but also includes an optically empty vitreous, chorioretinal atrophy, cataract, loss of peripheral vision, glial bands, eventual blindness, and an extinguished electroretinogram. In autosomal recessive foveal retinoschisis ${ }^{6}$ the lesion is limited to the macular area. The electroretinogram is normal. Autosomal dominant juvenile retinoschisis' sometimes affects the macula. In eight patients described by Yassur and colleagues ${ }^{7}$ three presented with foveal retinoschisis and five had macular pigmentary changes. Six patients had a normal single electroretinogram; the remainder had anormal a wave and a reduced $b$ wave that returned to above the baseline levels.

Hereditary retinoschisis without foveal retino-

Fig. 6 Right: A single-flash electroretinogram of the proband's father, measured after 20 minutes of dark adaptation, demonstrates $a$ normal a wave, a reduced $\mathrm{b}$ wave that returned to above baseline levels, and diminished oscillatory potentials. Left: $A n$ electroretinogram from a normal 35-year-old, measured after 20 minutes of dark adaptation.
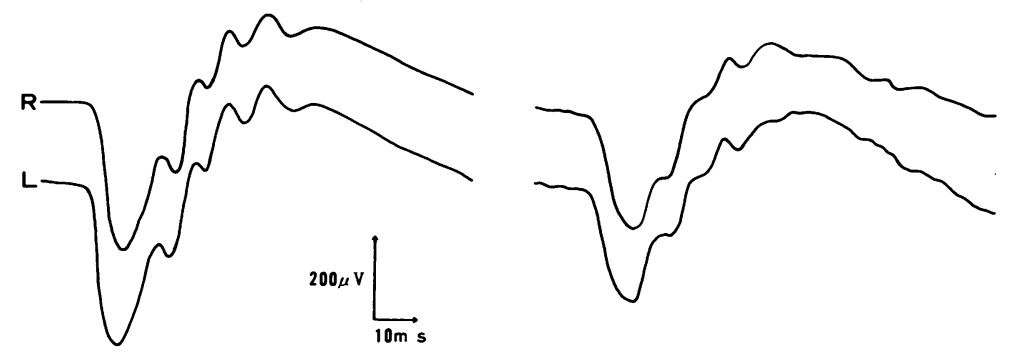
schisis is diagnostic for autosomal juvenile retinoschisis. ${ }^{\prime}$ Cibis ${ }^{1}$ described a family in which female monozygotic twins and their grandmother had peripheral retinoschisis without foveal retinoschisis. Their disorder was considered an autosomal form of retinoschisis with recessive or possibly even dominant transmission of inheritance. The father and daughter described here have retinoschisis without foveal retinoschisis. All members of the family live in a small, remote village. At least two consanguineous marriages have been recorded in the pedigree. Because the girl described in this report is also the product of a consanguineous marriage, the most likely mode of transmission in this family is autosomal recessive. The absence of foveal retinoschisis and the electroretinographic pattern displayed by the father and daughter also differentiate this condition from other forms of vitreoretinal dystrophy.

\section{References}

1 Cibis PA. Retinoschisis-retinal cysts. Trans Am Ophthalmol Soc 1965; 63: 417-53.

2 Deutman AF. Vitreoretinal dystrophies. In: Krill A, Archer DB, eds. Hereditary retinal and choroidal disease. New York: Harper and Row, 1977; 2: 1043-108.

3 Peachey NS, Fishmann GA, Derlacki DJ, Brigell MG. Psychophysiological and electroretinographic findings in $\mathrm{X}$-linked juvenile retinoschisis. Arch Ophthalmol 1987; 105: 513-6.

4 Gloria WU, Cotlier E, Brodie S. A carrier state of X-linked juvenile retinoschisis. Ophthalmic Pediatr Genet 1985; 5: 43-7.

5 Favre M. Apropos de deux cas de dégénérescence hyaloideoretinienne. Ophthalmologica 1958; 135: 604-9.

6 Lewis RA, Lee GB, Martonyi CL. Familial foveal retinoschisis. Arch Ophthalmol 1977; 95: 1190-6.

7 Yassur Y, Nissenkorn I, Kaffe S, Goodman RM. Autosomal dominant inheritance of retinoschisis. Am J Ophthalmol 1982; 94: $338-43$.

Accepted for publication 25 August 1988. 